Research Journal of Medical Sciences 13 (4): 76-81, 2019

ISSN: $1815-9346$

(C) Medwell Journals, 2019

\title{
Value of Platelet-Rich Plasma in Osteointegration of Tendon Graft in Anterior Cruciate Ligament Reconstruction
}

\author{
Midhat M. Mahdi and Hatem Halwas Jhale \\ Department of Orthopedic Surgery, College of Medicine, University of Basra, Basra, Iraq
}

\begin{abstract}
One of the challenges of Anterior Cruciate Ligament (ACL) reconstruction is to achieve secure graft attachment between the tendon and bone to allow early range of motion and activity and allowing an earlier return to unrestricted activity. To evaluate the role of Platelet Rich Plasma (PRP) in tendon-to-bone integration in the ACL reconstruction at (12+weeks) postoperative follow up by clinical, biomechanical and Magnetic Resonance (MR) imaging. A prospective randomized comparative study was done at Orthopedic Department of Basra General Hospital between June 2014 and October 2015. A twenty seven patients, male, active, young were divided into two groups (13) patients ACL reconstructions without PRP and (14) patients ACL reconstructions with PRP injection. Both groups were subjected to the same surgeon, same post operative rehabilitation program and same radiologist. The clinical (lysholum knee scaling score) results, (good and excellent score) were $8(57.2 \%)(\mathrm{p}=0.066)$. The stability tests results (anterior drawer test gradel) were 12 $(85.7 \%)(\mathrm{p}=0.000)$. Lachman test grade 1 were $12(85.7 \%)(\mathrm{p}=0.033)$, Pivot shift test grade 0 were $12(85.7 \%)$ $(\mathrm{p}=0.014)$. MRI findings showing signal intensity of fibrous interzone of femoral tunnel on Pdw-Fat Sat images grade $0(4) 28.6 \%(\mathrm{p}=0.057)$ and on T1W Fat Sat-Gad images grade $0(2) 14.3 \%(\mathrm{p}-0.067)$. But the range of knee motion arc for control group was best than those with PRP group $(\mathrm{p}<0.05)$ Post operative thigh girth difference in both groups was not significant $(\mathrm{p}<0.05)$. Post operative complications as pain, swelling seem to be more in PRP group than control group. These results were comparable to many other studies. Platelet Rich Plasma (PRP) is a safe procedure that has a beneficial effects in enhancement of graft-bone integration at $12+$ weeks postoperative follow up.
\end{abstract}

Key words: Basra General Hospital, adiologist, rehabilitation, magnetic resonance, platelet rich plasma, anterior cruciate ligament

\section{INTRODUCTION}

The outcomes of ACL reconstruction vary according to the graft choice, anatomical ACL reconstruction, secure graft fixation device (Tomita et al., 2001), pre and post operative rehabilitation. Using PRP in ACL repair in principle of that PRP is an autologus cell therapy containing many bioactive factors involving in wound healing and tissue repair as the platelets are a major source of growth factors and cytokines released from platelet degranulation (Anitua et al., 2004) facilitate the acute inflammatory phase which begins immediately upon release of the tourniquet due to bleeding from the surrounding bone could be ensuring efficient solid bone-graft integration (Castillo et al., 2011). The success of ACL reconstruction is dependent on 2 biological processes graft integration into the tibial and femoral tunnels. The earliest sign of osseous-graft integration is development of interface which defined by Weiler et al. (2001) and Petersen and Laprell (2000) as the fibrous interzone: (disorganized, highly cellular and highly vascular connective granulation tissue). Further healing, the FIZ tissue gets less cellular and vascular and there is an early development of Sharpey-like collagen fibers bridging the FIZ-bone interface. The FIZ-bone interface grows until reach a solid integration of the graft that is (the tissue between the graft and bone tunnel is poor in cells with little vascularity and rich collagen fiber) as in Fig. 1a. Maturation of the articular portion of the graft, at 6 weeks the graft is covered with thick synovial tissue, abundant capillaries and irregularly oriented collagen fibers, at 6 months the graft is thinly covered with synovium and is markedly less vascular and fibroblasts and collagen fibers are reoriented in a regular direction, by 1 year the graft is a thick ligamentous tissue resembling normal ACL, collagen fibers are organized in

Corresponding Author: Midhat M. Mahdi, Department of Orthopedic Surgery, College of Medicine, University of Basra, Basra, Iraq 
(a)
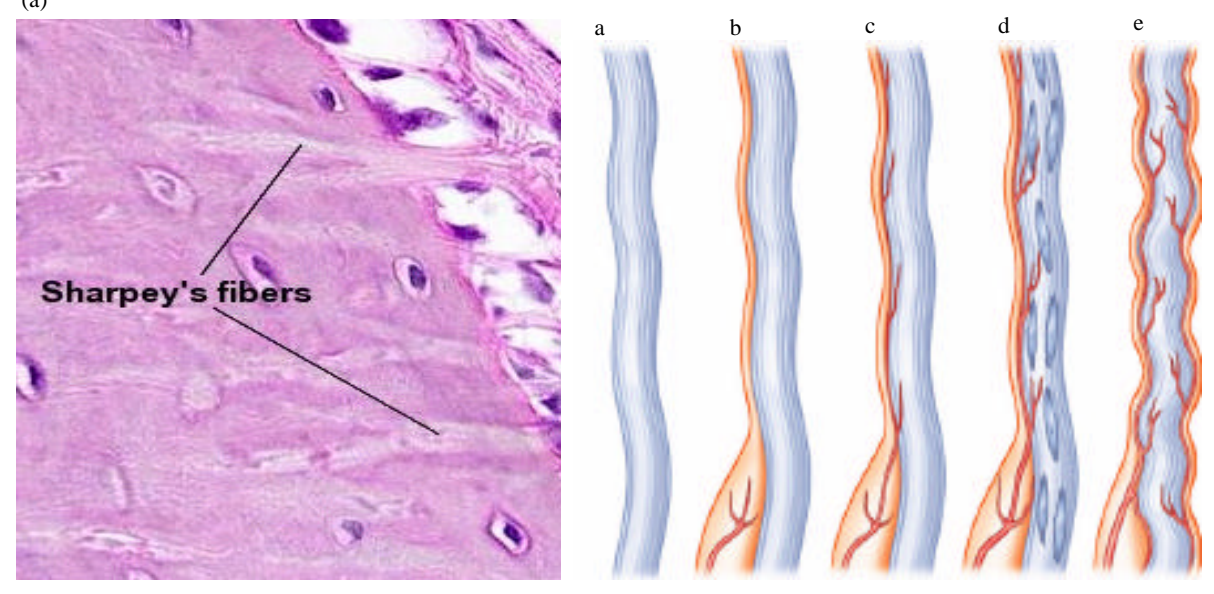

Fig. 1: a) Graft osteointegration and b) Tendon graft maturation; a) Cellular tendon, b) Revascularize graft, c) Remodeling d and e) Ligamentization

a high-frequency crimp pattern with spindle-shaped fibroblasts interspersed between collagen fibers (Rodeo et al., 1993) as in Fig. 1b.

\section{MATERIALS AND METHODS}

Patients and methods: A prospective randomized comparative study conducted at Orthopedic Department of Al-Basra General Hospital between June 2014 and October, 2015. Total numbers of 27 patients male, active, young (mean age $25.77 \pm$ years). Their mean body mass index $(24.41 \pm) \mathrm{kg} / \mathrm{m}^{2}$.

Inclusion criteria: Young, active patient with ACL rupture $<2$ years duration proved clinically and by MRI with anxious knee joint instability during daily activities, patient with full range of knee motion committed to preoperative physiotherapy with equal thigh girth in comparison to normal side.

Exclusion criteria: History of ACL rupture more than 2 year, limitation in range of motion with severe or gross muscle wasting patient with intraarticular knee fracture or around the knee joint, patients in PRP group unsuitable candidates for PRP donation (anemia, thrombocytopenia and hemodynamic ally unstable), patient with contraindications to surgery, MRI and those with cardiopulmonary dysfunction, Local or remote focus of infection, psychologically unstable patient. Patients for arthroscopic ACL reconstruction selected randomly and divided into two groups: group A: 13 patients without PRP. Group B: 14 patients with PRP in femoral tunnels and intraarticular at the end of surgery. The same surgeon and team group performed a single-bundle quadruple strand arthroscopic ACL reconstruction in all 27 patients using autologous hamstring tendons.

PRP preparation: The required amount of $P R P(6 \mathrm{~mL})$ was obtained by using Trima Accel Automated Blood Collection system. The Trima device is a continuous flow centrifuge. Around (100-150 mL) of PRP were collected from each patient, a pouch of $(6 \mathrm{~mL})$ of PRP was filled, sealed by sterile thermal sealer and given to the patient for injection. A total of 5.0-7.0 $\times 107$ platelets were yielded by machine after submitted with parameters. PRP can be used immediately or up to 5 days after collection, store at room temperature $\left(20-24^{\circ} \mathrm{C}\right)$ with continuous gentle agitation or may go up to $24 \mathrm{~h}$ without agitation.

Operative procedure: Supine position on operating table, pneumatic tourniquet, standard portals, graft harvested (semi tendinosis and gracilis tendon) and preparation tibial and femoral tunnels, anatomical position of graft, secure the graft with fixation device, deflation of tourniquet. At the end of surgery injection of inactivated PRP by using sterile spinal needle $(18 \mathrm{G} \times 3.5)(1.3 \times 88 \mathrm{~mm})$ that inserted in femoral tunnel between the graft and bone tunnel with help of arthroscopy then aspiration of $6 \mathrm{~mL} \mathrm{PRP} \mathrm{from} \mathrm{the} \mathrm{prepared} \mathrm{sealed} \mathrm{tube} \mathrm{with} 10 \mathrm{~mL}$ syringe, evacuation of irrigated fluid from knee joint then injection of $3 \mathrm{~mL}$ PRP in femoral tunnel after that we remove the spinal needle from tunnel toward the knee joint and inject the remaining $3 \mathrm{~mL}$ of PRP intra articularly as in Fig. 2.

At (12+weeks) postoperative both PRP and control groups were assessed by: Clinical Lysholm knee scoring scale, measurement of thigh girth, physical examination of 


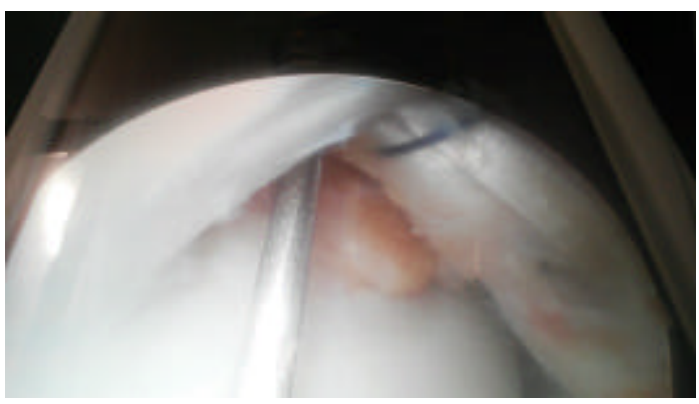

Fig. 2: Arthroscopic picture showing spinal needle that inserted in femoral tunnel between the graft and bone tunnel to start injection of PRP (Basra General Hospital)

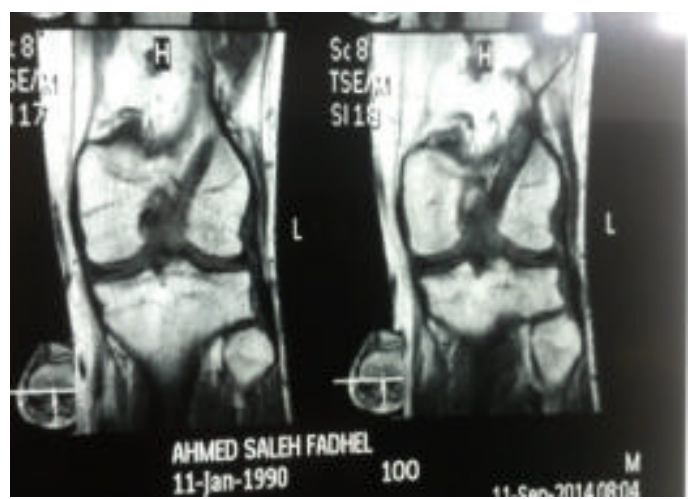

Fig. 3: PDW-Fat Sat MRI low singnal intensity (Grade 0) between graft and bone tunnel (Basra General Hospital)

Table 1: MR signal

\begin{tabular}{lll}
\hline MR signals & PDW-Fat Sat* & T1W-Fat Sat-Gad \\
\hline Grade 0 & Patellar tendon & Patellar tendon \\
Grade 1 & Skeletal muscle & Skeletal muscle \\
Grade 2 & >Skeletal Muscle & >Skeletal muscle< \\
& <fluid & synovial membrane \\
Grade 3 & Joint fluid & Synovial membrane \\
\hline
\end{tabular}

specific stability tests for ACL, MRI studies all images were done in Al-Basra Teaching Hospital Radiology Department and reviewed by the same radiologist. MRI parameters: \{PDW-Fat Sat and T1W-FatSat-Gad signal intensity compared (FIZ signal intensity) with knee structures in the same patient (Vogl et al., 2001) Table 1 (Fig. 3).

\section{RESULTS AND DISCUSSION}

Statistical analysis was performed using SPSS (statistical package for the social sciences) Version 16.0 Software proportions were compared by Chi-square test and Fisher's exact test. The significance level was set at
Table 2: Lysholm knee scoring scale

\begin{tabular}{llll}
\hline LKS knee score & Control & PRP & Total \\
\hline $65-83$ Fair & 9 & 6 & 15 \\
& $69.2 \%$ & $42.9 \%$ & $55.6 \%$ \\
84-90 Good & 4 & 6 & 10 \\
& $30.8 \%$ & $42.9 \%$ & $37.0 \%$ \\
$>90$ Excellent & 0 & 2 & 2 \\
& $0 \%$ & $14.3 \%$ & $7.4 \%$ \\
Total & 13 & 14 & 27 \\
& $100.0 \%$ & $100.0 \%$ & $100.0 \%$ \\
\hline
\end{tabular}

Table 3: Anterior drawer test

\begin{tabular}{llll}
\hline ADT grade $(\mathrm{mm})$ & Control & PRP & Total \\
\hline$<5$ & 1 & 12 & 13 \\
& $7.7 \%$ & 85.7 & $48.1 \%$ \\
$5-10$ & 10 & 2 & 12 \\
& $76.9 \%$ & $14.3 \%$ & $44.4 \%$ \\
$>10$ & 2 & & \\
& $15.4 \%$ & $0 \%$ & $7.4 \%$ \\
Total & 13 & 14 & 27 \\
& $100.0 \%$ & $100.0 \%$ & $100.0 \%$ \\
\hline
\end{tabular}

Table 4: Lachman test

\begin{tabular}{llll}
\hline Lachman test grade (mm) & Control & PRP & Total \\
\hline$<5$ & 6 & 12 & 18 \\
& $46.2 \%$ & $85.7 \%$ & $66.7 \%$ \\
$6-10$ & 7 & 2 & 9 \\
& $53.8 \%$ & $14.3 \%$ & $33.3 \%$ \\
Total & 13 & 14 & 27 \\
& $100.0 \%$ & $100.0 \%$ & $100.0 \%$ \\
\hline
\end{tabular}

Table 5: Pivot shift test

\begin{tabular}{llll}
\hline Pivot shift test grade & Control & PRP & Total \\
\hline Absent & 5 & 12 & 17 \\
& $38.5 \%$ & $85.7 \%$ & $63.0 \%$ \\
Pivot glide & 8 & 2 & 10 \\
& $61.5 \%$ & $14.3 \%$ & $37.0 \%$ \\
Total & 13 & 14 & 27 \\
& $100.0 \%$ & $100.0 \%$ & $100.0 \%$ \\
\hline
\end{tabular}

$\mathrm{p}<0.05$. Table 2 (Lysholm knee scoring scale) (Good and Excellent scores) for PRP group were 8 and for control groups were 4. (Fair score) for PRP patients were 6 and for control groups were 9. $(\mathrm{p}=0.066$ (significant) and Table 3. Specific ACL stability tests of knee (Anterior Drawer Test (ADT grades in $\mathrm{mm}$ ) $<5 \mathrm{~mm}$ difference (grade 1) for PRP group were 12 and for control group was 1 patient $\mathrm{p}=0.000$ (significant).

Table 4 Lachman test: The $<5 \mathrm{~mm}$ difference for PRP group (grade 1) were 12 patients while for control group were 6 patients. More than $5 \mathrm{~mm}$ difference (grade 2) for PRP group 2 patients in comparison to control group 7. $\mathrm{p}=0.033$ (significant).

Table 5 pivot shift test: absent (grade 0) for PRP group were 12 patients while for control group were 5 patients. Pivot shift test grade 1 (Pivot glide) for PRP group were 2 patients and in control group were 8 patients $\mathrm{p}=0.014$ (significant).

Table 6: Signal intensity of fibrous interzone of femoral tunnel on MRI signal grade PDW-Fat Sat (coronal proton density-weighted images with spectral-Fat 
Res. J.Med.Sci., 13 (4): 76-81, 2019

\begin{tabular}{llll}
\multicolumn{4}{l}{ Table 6: Signal intensity of fibrous interzone of femoral tunnel } \\
\hline MRI signal grade PDW-Fat & Control & PRP & Total \\
\hline 0 & 0 & 4 & 4 \\
& $0 \%$ & $28.6 \%$ & $14.8 \%$ \\
1 & 13 & 10 & 23 \\
& $100.0 \%$ & $71.4 \%$ & $85.2 \%$ \\
2 & 0 & 0 & 0 \\
& $0 \%$ & $0 \%$ & $0 \%$ \\
3 & 0 & 0 & 0 \\
Total & $0 \%$ & $0 \%$ & $0 \%$ \\
& 13 & 14 & 27 \\
& $100.0 \%$ & $100.0 \%$ & $100.0 \%$
\end{tabular}

Table 7: MRI signal grade T1W-Fat Sat-Gad

\begin{tabular}{llll}
\hline MRI signal grade T1W_Fatsat-Gad & Control & PRP & Total \\
\hline 0 & 0 & 2 & 2 \\
& $0 \%$ & $14.3 \%$ & $7.4 \%$ \\
1 & 11 & 12 & 23 \\
& $84.6 \%$ & $85.7 \%$ & $85.2 \%$ \\
2 & 2 & 0 & 2 \\
& $3.4 \%$ & $0 \%$ & $7.4 \%$ \\
\multirow{2}{*}{ Total } & $15.4 \%$ & 0 & 0 \\
& 0 & $0 \%$ & $0 \%$ \\
& $0 \%$ & 14 & 27 \\
& 13 & $100.0 \%$ & $100.0 \%$ \\
\hline
\end{tabular}

Table 8: Range of knee motion

\begin{tabular}{llll}
\hline Range of knee motion in degree & Control & PRP & Total \\
\hline$>130$ & 4 & 3 & 7 \\
& $30.8 \%$ & $21.4 \%$ & $25.9 \%$ \\
$120-130$ & 8 & 7 & 15 \\
& $61.5 \%$ & $50.0 \%$ & $55.6 \%$ \\
$110-119$ & 1 & 2 & 3 \\
& $7.7 \%$ & $214.3 \%$ & $311.1 \%$ \\
$100-109$ & 0 & 2 & 2 \\
& $0.0 \%$ & $214.3 \%$ & $27.4 \%$ \\
Total & 13 & 14 & 27 \\
& $100.0 \%$ & $14100.0 \%$ & $27100.0 \%$ \\
\hline
\end{tabular}

saturation) in PRP group 4 patients were grade 0 and 10 patients were grade 1 while in control group no patient had grade 0 and 13 patients had grade 1 signal intensity. $\mathrm{p}=0.057$ ( significant).

Table 7 MRI signal grade T1W-Fat Sat-Gad (T1-weighted images with spectral fat-saturation with gadolinium). In PRP group 2 patients were grade 0 and 12 patients were grade 1 but no 2 or grade 3 patients. In control group no patient had grade 0 and 11 patient were grade 1 and 2 patients were grade $2 . p=0.067$ (marginal significant).

Table 8 range of knee motion arc in (degree) postoperative was measured by goniometer. Patients with $\mathrm{ROM}<120^{\circ}$ for control group were 1 in comparison to PRP group patients were 4. $\mathrm{p}=0.075$ (not significant).

Table 9 postoperative complications distribution pain in PRP group were 4 patients in comparison to control were 2 patients, knee swelling in PRP group were 5 patients while in control group were 2 , infection in PRP group were 2 patients while in control group were 1 patient, knee stiffness in PRP group were 2 patients while no stiffness in control group.
Table 9: Post operative complications distribution

\begin{tabular}{lcccc}
\hline $\begin{array}{l}\text { Post operative } \\
\text { complications }\end{array}$ & $\begin{array}{l}\text { PRP } \\
\text { group }\end{array}$ & Percentage & $\begin{array}{c}\text { Control } \\
\text { group }\end{array}$ & Percentage \\
\hline Postoperative pain & 4 & 28.50 & 2 & 15.38 \\
Knee swelling & 5 & 35.70 & 2 & 15.38 \\
infection & 2 & 14.28 & 1 & 07.69 \\
D.V.T. & 0 & 0 & 0 & 0 \\
Neurovascular injury & 0 & 0 & 0 & 0 \\
knee stiffness & 2 & 14.28 & 0 & 0 \\
\hline
\end{tabular}

Anterior cruciate ligament injury is a common knee sport injury and at the present time ACL repairs has increasingly done worldwide. The success of ACL reconstruction is depends on graft integration into bone tunnels. Platelet Rich Plasma (PRP) is a probable factor for enhancement of osseous soft-tissue graft incorporation and prevention of graft failure that might help the patients for early return to pre injury activity levels and early return to sport participation (Woo et al., 1998). The earliest sign of osseous-graft integration is development of interface the Fibrous Interzone (FIZ) which consists of disorganized, highly cellular and highly vtascular connective granulation tissue. A solid integration of the graft means (the tissue between the graft and bone tunnel is poor in cells with little vascularity and rich in collagen fiber). In this study usage of hamstring tendon grafts with "suspensory" fixation mechanism (Endobutton) resulting in tendon integration by indirect insertion. Indirect insertion comprises three layers: tendon, Sharpey's fibers and bone (Nebelung et al., 2003). In this study we choose a ( 12 weeks) period after surgery to perform evaluation of two groups regarding clinical, knee stability specific tests and MRI imaging. This choice was based on some studies that show the tendon-to-bone attachment occurs by 12th week after surgery and considered the best time for appreciation changes in signal intensity in MRI (Hartigan et al., 2012). There is a correlation between MR signal intensity and histological graft maturation was established by Weiler et al. (2001).

Patients were randomly selected, minimum with mean age $(25.77 \pm$ year). Older age may negatively affect the outcome (Magnussen et al., 2012). However, there is no evidence that gender or age affects platelet count or growth factor concentration in PRP (Ventura et al., 2005). In this study all patients selected were males, mean body mass index $(24.41 \pm) \mathrm{kg} / \mathrm{m}^{2}$ some studies revealed that female gender and higher body mass index may adversely affect the outcome (Sanchez et al., 2012). For all our patients whom subjected to ACL reconstruction the hamstring tendon graft size were $8+\mathrm{mm}$ and above and no one of them reconstructed with graft size $<8 \mathrm{~mm}$. Some recent clinical studies demonstrate a higher failure rate for hamstring tendon $\mathrm{ACL}$ reconstructions when the diameter 
of the ACL replacement graft is $<8 \mathrm{~mm}$ (Dunn et al., 2010). In this study patients were evaluated clinically by (Lysolum Knee Scoring Scale) for PRP group (Good and excellent scores) were $8(57.2 \%)$ and for control groups (good and excellent scores) were $4(30.8 \%)$. The $p=0.066$ (significant). Accordingly, the good clinical outcome results were in favor of PRP group. Several studies reported clinical outcomes after ACL reconstructive surgery with or without PRP augmentation, focusing on the short-term outcome with follow up reported from 6 months to 2 years. None of them showed any statistical inter-group difference. Only Magnussen et al. (2012) in a comparative retrospective trial observed a lower swelling reaction and pain in the PRP group that significant below 8 weeks after operation (Ventura et al., 2005). In this study specific (ACL stability tests of knee): anterior drawer test ( $<5 \mathrm{~mm}$ difference (grade 1) were $12(85.7 \%$ ) while for control group patients (grade 1) were $1(7.7 \%) \mathrm{p}=0.000$ (significant) this resulted in a significant stability in favor of PRP group. Lachman test grade $(\mathrm{mm})<5 \mathrm{~mm}$ difference ((grade 1) were 12 (85.7\%) while for control group patients (grade 1) were 6(46.2\%) $p=0.033$ (significant). Pivot Shift test grade pivot shift test for PRP group absent (grade 0 ) were $12(85.7 \%)$ patients while for control group patients (grade 0 ) were $5(38.5 \%) \mathrm{p}=0.014$ (significant). Specific stability tests for ACL reconstruction seem to be solid for PRP group in comparison to control group probably because of a good graft incorporation in bone tunnel. Though the specific ACL stability tests that done vulnerable to inter-observer variability and difficult assessment in un relaxed patient, so, preferable done under general anesthesia. In this study we compared the signal intensity of the FIZ on MR imaging among the patient groups A, B. MRI signal grade PDW-Fat Sat for patients with PRP group (grade 0 ) was $4(28.6 \%)$ while in patients control group was $O(0 \%)$ that had (grade 0 ) signal intensity $\mathrm{p}=0.057$ (significant). MRI signal grade $\mathrm{T} 1 \mathrm{~W}-$ Fatsat-Gad patients with PRP group (grade 0 ) was $2(14.3 \%)$ while in patients control group was $0(0 \%)$ that had (grade 0) signal intensity $\mathrm{p}=0.067$ (marginal significant). MR images with low signal intensity, similar to patellar tendon (grade 0), on PDW-Fat Sat and T1W FatSat- Gad images for PRP group means the FIZ with low cellularity, low vascularity and rich collagen (solid integration). In two MRI scan signals, Rough estimation of fibrouse interzone revealed that PRP group had a good collagen fibers and a good graft-bone incorporation in comparison to patients reconstructed ACL without PRP, these results of this study was promising a positive outcomes to achieve a solid integration in ACL reconstructions. Other agonist studies to this study like Ventura et al. (2005) studied ACL treatment with PRP in humans and his studies revealed an enhancing effect in the healing process (Ventura et al., 2005). By Rupreht et al. (2013) were documenting PRP benefit in the bony tunnel/graft area. Among the studies documenting no beneficial effects of PRP administration, the (Silva and Sampaio, 2009) who evaluated the effect of PRP augmentation in graft integration within the femoral tunnels. Another study by Vogrin et al. (2010) investigated the vascularization at the interface between bone tunnels and graft and along the intra-articular portion of the graft: a superior vascularization was found in the PRP group only at 4-6 weeks with MRI but not at 10-12 weeks (Vogrin et al., 2010). Range of knee motion (degree) postoperatively, for patients with range of knee movement $>120^{\circ}$ for control group were $1(7.7 \%)$ in comparison to $\mathrm{PRP}$ group patients were $4(28.6 \%)$ $p=0.075$ (not significant). As shown that range of knee motion is more in control group than of PRP group probably because of pain intolerance. In this study postoperative complication: postoperative pain in PRP group were $4(28.5 \%)$ patients in comparison to control were $2(15.38 \%)$ patients were well responded to non potent analgesia, knee swelling in PRP group were 5 $(35.7 \%)$ patients while in control group were $2(15.38 \%)$, infection in PRP group were 2(14.28\%) patients while in control group were $1(7.69 \%)$ the infection of two of them superficial infection treated by antibiotics and one of them deep infection treated by aspiration and parenteral antibiotic all of them completely resolved. Knee stiffness in PRP group were $2(14.28 \%)$ patients while in control group were $0(0 \%)$ for both of them were treated by cautious manipulation under general anesthesia with programmed physiotherapy. postoperative complication seems to be more in PRP group may be due to intense inflammatory reaction.

\section{CONCLUSION}

Platelet Rich Plasma (PRP) s a safe procedure regarding preparation, no risk of disease transmission or systemic immunological reactions. The PRP has a beneficial effects that enhance graft-bone integration at 3 months postoperative which evaluated by MRI images. Clinical and biomechanical evaluation were demonstrated that PRP had an encouraging outcomes that help in early recovery and probably returns to pre injury state. The PRP had undesirable effects in patients with ACL reconstruction augmented with PRP in concerns to range of knee movements, postoperative swelling and knee stiffness which may be due to pain intolerance that accompanied the intense inflammatory reactions. 


\section{REFERENCES}

Anitua, E., I. Andia, B. Ardanza, P. Nurden and A.T. Nurden, 2004. Autologous platelets as a source of proteins for healing and tissue regeneration. Thrombosis Haemostasis, 91: 4-15.

Castillo, T.N., M.A. Pouliot, H.J. Kim and J.L. Dragoo, 2011. Comparison of growth factor and platelet concentration from commercial platelet-rich plasma separation systems. Am. J. Sports Med., 39: 266-271.

Dunn, W.R., K.P. Spindler and M. Consortium, 2010. Predictors of activity level 2 years after Anterior Cruciate Ligament Reconstruction (ACLR) a Multicenter Orthopaedic Outcomes Network (MOON) ACLR cohort study. Am. J. Sports Med., 38: 2040-2050.

Hartigan, E.H., J. Zeni Jr, S.D. Stasi, M.J. Axe and L. Snyder-Mackler, 2012. Preoperative predictors for noncopers to pass return to sports criteria after ACL reconstruction. J. Appl. Biomech., 28: 366-373.

Magnussen, R.A., J.T.R. Lawrence, R.L. West, A.P. Toth and D.C. Taylor et al., 2012. Graft size and patient age are predictors of early revision after anterior cruciate ligament reconstruction with hamstring autograft. Arthroscopy J. Arthroscopic Relat. Surg., 28: 526-531.

Nebelung, W., R. Becker, D. Urbach, M. Ropke and A. Roessner, 2003. Histological findings of tendon-bone healing following anterior cruciate ligament reconstruction with hamstring grafts. Arch. Orthop. Trauma Surg., 123: 158-163.

Petersen, W. and H. Laprell, 2000. Insertion of autologous tendon grafts to the bone: A histological and immunohistochemical study of hamstring and patellar tendon grafts. Knee Surg. Sports Traumatology Arthroscopy, 8: 26-31.

Rodeo, S.A., S.P. Arnoczky, P.A. Torzilli, C. Hidaka and R.F. Warren, 1993. Tendon-healing in a bone tunnel: A biomechanical and histological study in the dog. J. Bone Joint Surg., 75: 1795-1803.

Rupreht, M., V. Jevtic, I. Sersa, M. Vogrin and M. Jevsek, 2013. Evaluation of the tibial tunnel after intraoperatively administered platelet-rich plasma gel during anterior cruciate ligament reconstruction using diffusion weighted and dynamic contrast-enhanced MRI. J. Magn. Reson. Imaging, 37: 928-935.
Sanchez, M., I. Andia, E. Anitua and P. Sanchez, 2012. Platelet Rich Plasma (PRP) Biotechnology: Concepts and Therapeutic Applications in Orthopedics and Sports Medicine. In: Innovations in Biotechnology, Agbo, E.C. (Ed.). Intech Open Access, London, UK., ISBN:978-953-51-0096-6, pp: 953-978.

Silva, A. and R. Sampaio, 2009. Anatomic ACL reconstruction: Does the platelet-rich plasma accelerate tendon healing?. Knee Surg. Sports Traumatology Arthroscopy, 17: 676-682.

Tomita, F., K. Yasuda, S. Mikami, T. Sakai and S. Yamazaki et al., 2001. Comparisons of intraosseous graft healing between the doubled flexor tendon graft and the bone-patellar tendon-bone graft in anterior cruciate ligament reconstruction. Arthroscopy J. Arthroscopic Relat. Surg., 17: 461-476.

Ventura, A., C. Terzaghi, E. Borgo, C. Verdoia and M. Gallazzi et al., 2005. Use of growth factors in ACL surgery: Preliminary study. J. Orthop. Traumatology, 6: 76-79.

Vogl, T.J., J. Schmitt, J. Lubrich, K. Hochmuth and T. Diebold et al., 2001. Reconstructed anterior cruciate ligaments using patellar tendon ligament grafts: Diagnostic value of contrast-enhanced MRI in a 2-year follow-up regimen. Eur. Radiol., 11: $1450-1456$.

Vogrin, M., M. Rupreht, D. Dinevski, M. Haspl and M. Kuhta et al., 2010. Effects of a platelet gel on early graft revascularization after anterior cruciate ligament reconstruction: A prospective, randomized, double-blind, clinical trial. Eur. Surg. Res., 45: 77-85.

Weiler, A., G. Peters, J. Maurer, F.N. Unterhauser and N.P. Sudkamp, 2001. Biomechanical properties and vascularity of an anterior cruciate ligament graft can be predicted by contrast-enhanced magnetic resonance imaging: A two-year study in sheep. Am. J. Sports Med., 29: 751-761.

Woo, S.L., J.K. Suh, I.M. Parsons IV, J.H. Wang and N. Watanabe, 1998. Biologic intervention in ligament healing: Effect of growth factors. Sports Med. Arthroscopy Rev., 6: 74-82. 